\title{
From cognitive control to creativity: The conflict resolution of surrealistic imagery
}

\author{
Manuela Ruzzoli ${ }^{1,2}$, Aoife McGuinness ${ }^{1}$, Luis Morís Fernández ${ }^{1,3}$, Salvador Soto- \\ Faraco $^{1,4}$
}

1. Multisensory Research Group, Center for Brain and Cognition, Universitat Pompeu Fabra, 08018 Barcelona, Spain.

2. Centre for Cognitive Neuroimaging, Institute of Neuroscience and Psychology, University of Glasgow, G12 8QQ, Glasgow, UK.

3. Departamento de Psicología Básica, Universidad Autónoma de Madrid, Madrid, Spain

4. Institució Catalana de Recerca i Estudis Avançats (ICREA), Barcelona, Spain.

Corresponding author: Manuela Ruzzoli (manuela.ruzzoli@gmail.com). Centre for Cognitive Neuroimaging, Institute of Neuroscience and Psychology, University of Glasgow, 62 Hillhead Street, G12 8QQ, Glasgow, UK.

Running head: Cognitive Control \& Surrealistic imagery 


\begin{abstract}
Surrealistic imagery juxtaposes common visual elements to create un-ordinary associations with semantically conflicting representations. We hypothesize that these images engage the conflict processing network that has been described in cognitive neuroscience theories, and probed with laboratory protocols such as the Stroop or Flanker tasks. We addressed this hypothesis by measuring mid-frontal Theta power using EEG while participants watched surrealistic images through a social media-like interface. Surrealistic images, compared to controls, produced a significant Theta power increase, as predicted from cognitive conflict theory. We also found increased memory for surrealistic images one week after exposure, compared to the controls. These findings provide evidence for the surrealistic artistic strategy to effectively engage viewer's cognitive control and boost memorability. In these studies, we provide validation of cognitive theories in real-life scenarios and insights regarding the use of neural correlates as effectiveness metrics in creative communication, as well as the arts.
\end{abstract}

Keywords: EEG, Theta Power, Oscillations, Cognitive Conflict, Memory, Creative Communication, Surrealism 


\section{Introduction}

Surrealistic art techniques, an outgrowth of the early 20th-century artistic movement, deliberately defy reason by the juxtaposition of incongruent elements. These techniques have become a persuasive communication strategy in advertising, film and visual arts (Homer \& Kahle, 1986; Lee \& Schumann, 2004). Despite the pervasive use of this strategy to attract attention and increase memorability, the brain processes associated with looking at surrealistic images are unknown. Here, we address the hypothesis that surrealist imagery may engage the conflict processing brain network.

The conflict monitoring theory (Botvinick, Braver, Barch, Carter, \& Cohen, 2001; Inzlicht, Shenhav, \& Olivola, 2017; Shenhav, Botvinick, \& Cohen, 2013; Shenhav, Cohen, \& Botvinick, 2016) proposes that the anterior cingulate cortex (ACC), a frontal-medial brain structure, is responsive to the presence of conflict in information processing; being conflict defined as "the simultaneous activation of incompatible and competing representations" (Botvinick, Cohen, \& Carter, 2004). The function of the ACC is to trigger adjustments in cognitive control to flexibly allocate resources via attention (Botvinick et al., 2001, 2004), in order to minimize or resolve the conflict. This helps us behave adaptively in a changing, demanding environment.

Cognitive conflict is typically tested in laboratory protocols employing the Stroop task (Stroop, 1935), the Simon task (Simon \& Small, 1969) or the Flanker task (Eriksen \& Eriksen, 1974) which notably produce consistent findings (Macleod, 1991). Conflict between stimuli or potential response options are associated with increases in BOLD responses in the ACC (measured with fMRI) (Braver, Barch, \& Gray, 2001; Kerns et al., 2004) and in Theta oscillatory activity (4-8 Hz) over mid-frontal sensors (measured with EEG or MEG) (Cavanagh, Figueroa, Cohen, \& Frank, 2012; Cohen, 2014). In the present study, we tested the hypothesis that visual imagery that contains incongruent meanings, such as that used in advertising, will trigger responses in line with the conflict brain network. Conflict response will be reflected in frontal-medial Theta power $(4-8 \mathrm{~Hz})$, compared to control images that do not contain incongruity.

Furthermore, we assess the impact of conflict on subsequent memory by introducing a recall task one week after exposure to the images. We address the specific hypothesis regarding the memorability of surrealist imagery, implicit in advertising. Cognitive neuroscience has suggested a relationship between visual memory capacity and semantic consistency (see Brady, Konkle, \& Alvarez, 2011), whereby objects that are inconsistent with the surrounding scene are remembered more (see also Friedman, 1979; Krebs, Boehler, De Belder, \& Egner, 2015). The impact of surrealistic ads on memory has been only tested in a seminal study by Homer \& Kahle (1986) using a free-recall test. They found that participants who saw surrealistic ads were less inclined to errors, compared to a control group, thus supporting the proposal that surrealistic images strengthen memory. To the best of our knowledge, the only neuroimaging study looking at brain responses to surrealistic imagery in advertising (Mostafa, 2013), showed greater activation in the anterior cingulate cortex (ACC), amongst other areas. Although the advertising industry often exploits conflict as a creative device to boost memorability, this seems to be the first study that addresses this assumption from a neuroscientific perspective, combining electrophysiological and behavioral responses. 


\section{Methods}

\section{Pre-registered protocol}

The initial hypotheses, procedure and analytical pipeline were pre-registered before data collection in the OSF platform (https://osf.io/xa7rv/). Here we present the results obtained in the pre-registered analyses, plus further exploratory analyses carried out as follow-ups (specified below).

\section{Participants}

We recorded data from 31 healthy participants ( 15 females) aged between 18 - 34 years $(22 \pm 3)$, with normal or corrected-to-normal vision. Data from two participants were excluded because of a technical problem in EEG data storing $(\mathrm{N}=1)$, and because of a failure to comply with the instructions in the memory task $(\mathrm{N}=1)$. After EEG artefact rejection, data from two further participants were excluded because less than 30 artefact-free trials were left for analysis. A total of 27 participants were thus included in the analyses. Only for the Theta peak analysis, data from one participant were excluded because no peak in the Theta range could be found in the control condition, leaving a final sample of 26 participants for that particular analysis. The experiment was run following the Declaration of Helsinki and approved by the ethics committee CIEC Parc de Mar (Universitat Pompeu Fabra, Barcelona, Spain). Participants gave written informed consent before participating in the study and received a compensation of 10 euro/hour for their time.

\section{Apparatus \& stimuli}

The experiment was designed and ran using Psychtoolbox (Brainard, 1997) on Matlab R2016b. Visual stimuli were presented through a CRT monitor (1024 x 768 pixels), with a refresh rate of $60 \mathrm{~Hz}$ and 32-bit color resolution. A set of 120 incongruent images were selected from image directories such as Pinterest and Google Image Search, with the following search queries: 'Creative Advertising', 'Smart Ads', 'Innovative Advertising', and 'Surrealist Ads'. One hundred and five of the selected images were advertisements, and 15 were similar artistic images. The selection of the set of incongruent images was initially based on the author's subjective evaluation of whether it could be considered 'out of the ordinary' in terms of the semantics or visual content and whether it contained two or more interacting objects that are usually semantically unrelated (see Figure 1.A for an example).

A set of matching control images, which did not contain any obvious semantic incongruity, was selected for each of the 120 incongruent images based on visual similarity. The tools used to find visually similar images were 'Google Reverse Image Search' and the website 'Yandex' (https://yandex.com/). Reverse image search is a content-based image retrieval (CBIR) technique that involves providing the system with an image that it will then formulate its search upon. All images were edited in Photoshop (version CS6) to remove text and any branding (e.g., logos) if present.

All 240 images initially selected with the methods above were then subjected to a survey to validate the levels of visual incongruity for each image by independent judges. The survey consisted of determining the degree of agreement of two statements regarding the categories of originality and synthesis from Smith et al.' study (Smith et al., 2007), which can be considered an objective way to determine image incongruity. We asked the participants to score each image from 1 (total disagreement) to 7 (full agreement) according to the following statements: 
1. The image is out of the ordinary.

2. The image connects objects that are usually unrelated.

To shorten the duration of the survey for each participant, we divided the whole set of 240 images into three equivalent 80-image surveys randomly distributed online via the Qualtrics survey platform (Qualtrics, Provo, UT, 2018). We obtained data from a total of 122 respondents ( $N=48$, 38, and 36, for Surveys 1, 2, and 3, respectively), who were not involved in the subsequent tests.

The results of the surveys were then used to discard images rated with low levels of incongruity from the incongruent experimental set, as well as images rated with high levels of incongruity from the control set. Incongruent images with a mean rating less than 4 were discarded, and control images with a mean rating of 4 and above were discarded. The top 100 images in each condition were then selected (200 in total) as the experimental image set.

The paradigm also included filler images based on trending topics on Twitter (circa March 2018) in various locations worldwide. A random mix of images related to current affairs, pop culture, sports and entertainment were chosen to mimic social media news feeds. We decided to include fillers because we wanted to create a realistic social media context. In addition, we assumed that interspersed filler images would dilute the contrast between incongruent and control stimuli and, would, by increasing the image set, make the subsequent memory task more difficult. A total of 250 images (100 incongruent +100 controls +50 fillers) were used for the EEG experiment.

For the test during the EEG recording, the 100 incongruent images and their corresponding 100 controls were split into two equivalent sets (counterbalanced across participants) so that only one image of each pair (either the incongruent or the control) appeared to each participant, but all images appeared the same number of times across the whole experiment. In addition to the 50 control and 50 incongruent images, each set also included all the 50 fillers. Each participant viewed only one image set during the EEG recording and was tested using all images (from both sets) in the subsequent memory test.

\section{EEG recording}

During the exposure session, EEG was continuously recorded through 16 electrodes using a portable EEG system (Enobio, Neuroelectrics, Barcelona, Spain) with a custom electrode montage, focused around the mid-frontal area (see Figure 1). Horizontal and vertical electrooculograms (hEOG and vEOG) were recorded from two additional electrodes placed at the outer canthus and under the right eye, respectively. Two additional electrodes were placed over the left and right mastoids for off-line re-referencing. Online reference electrode and ground were clipped onto the left earlobe.

In the spirit of ecological generalisation underlying this study, we attempted to test our hypotheses under close to realistic conditions to improve the feasibility of applying such measurements outside the laboratory (Matusz, Dikker, Huth, \& Perrodin, 2019; Soto-Faraco et al., 2019). This is why we used a wearable EEG headset that makes it more agile and applicable to real-world testing. This 16 electrode montage proved enough to measure mid-frontal Theta power (Figure 1). The theory-driven approach adopted here helped to narrow down on specific scalp sites and compensate for the relatively sparse spatial sampling. 


\section{Experiment 1: EEG test}

The EEG experiment was performed in a dimly lit room and lasted about 1 hour. Each participant viewed 150 images (50 incongruent, 50 control and 50 filler) in random order. Participants were asked to decide whether they thought the image was directed to a male or female audience, to proceed to the next image. This task was orthogonal to the goals of the study and was devised merely to ensure that participants looked at each image closely during the EEG phase. No specific analyses were conducted on this response.

Participants rested their hands palm down, with the index fingers positioned on the response keys of the computer keyboard (' $z$ ' and ' $m$ ' for left- and right-hand responses, respectively). At the beginning of each trial, a frontal view of a smartphone displaying the typical loading wheel on the screen (mimicking a social media load screen, where participants would browse images amongst other information related to current news, in a self-paced manner) was presented for $1500 \mathrm{~ms}$, which served as fixation. Next, a randomly chosen incongruent, control or filler image appeared on the smartphone screen. After $2000 \mathrm{~ms}$, a male and female icon appeared on either side of the image, signalling to participants that they could respond using the ' $\mathrm{z}$ ' and ' $\mathrm{m}$ ' keys, corresponding to a gender. The position of the male and female icons was randomized across trials so that participants could not prepare their response in advance, to avoid motor response contamination in the EEG signal. The following trial started after a randomly jittered period ( 800 to $1000 \mathrm{~ms}$ ) following response. Each participant ran a total of 5 experimental blocks of 30 trials each.

\section{Experiment 2: Memory test}

The primary aim for the memory test was to address whether incongruent images were spontaneously remembered better than controls. Also, overall performance in the memory test would work as a reality check, ensuring that participants looked at the images during the EEG experiment. We invited participants to come back to the lab one week after the exposure phase with EEG recordings and to run a $2 \mathrm{AFC}$ (two-alternative forced-choice) task. Before or during the EEG experiment, participants were not informed that they would be asked to perform a memory test one week later. In the memory task, participants were given to choose which of two images presented side-by-side they remembered seeing during the study session, one week before. The memory test comprised of 200 images (100 pairs) in total. That is, the full sets of congruent and incongruent images, including the 50 incongruent (and 50 congruent) images previously seen, randomly paired with the 50 incongruent (and 50 congruent) images which had not been seen during the EEG recording. All images were transformed into black and white to encourage participants to rely on their conceptual memory of images, rather than perceptual features (Dzulkifli \& Mustafar, 2013). Participants rested their hands palm down, with the index fingers positioned on the response keys of the computer keyboard (' $z$ ' and ' $m$ ' for left- and right-hand responses, respectively). A fixation cross appeared in the middle of the screen, along with the image pairs $(1000 \mathrm{~ms})$ presented laterally. Participants were instructed to respond as fast as possible, indicating which one of the two presented images they remembered having seen during the EEG experiment. Once the images disappeared, the fixation-cross remained until participants pressed either the ' $z$ ' or ' $m$ ' key, corresponding to the image on the left and the right-hand side of the fixation. The position of the seen image in the pair (correct response) was counter-balanced. The next pair of images appeared $1000 \mathrm{~ms}$ after the response (Figure 1B). 


\section{Data pre-processing}

EEG data was high-pass filtered from 0.5 and low-pass from $40 \mathrm{~Hz}$ (Butterworth filters of order 2 and 8 respectively), with an additional notch filter at $50 \mathrm{~Hz}$. Although our initial intention was to re-reference the signal to the averaged signal from mastoid electrodes, due to excessive recording noise in the mastoid electrodes we decided to proceed without re-referencing. This decision was taken based on data quality and prior to any further data analysis. Data were segmented into epochs starting from $-1000 \mathrm{~ms}$ up to $2000 \mathrm{~ms}$ with respect to image onset time. Because the target images were presented on the screen for 2 seconds and we allowed the participants to explore the scene visually, the EEG signal was largely contaminated by artefacts from horizontal and vertical eye movements. Therefore, an independent component analysis (ICA) using the FieldTrip toolbox (Oostenveld, Fries, Maris, \& Schoffelen, 2011) was run for artefact removal, since the rejection of the entire trials would have left too few trials for analysis. Components were visually inspected and those corresponding to visual artefacts were removed. On average, two ICA components were removed per participant. All trials were visually inspected afterwards to assess the quality of the removal. As per the pre-registration document (https://osf.io/wftd7/), our focus was on Theta power (4-8 Hz) at mid-frontal location (Fz, Cz, $\mathrm{FCz})$ in the time window between stimulus onset $(0 \mathrm{~ms})$ to $2000 \mathrm{~ms}$ post-stimulus. As baseline, we considered the time window between $-750 \mathrm{~ms}$ and $-250 \mathrm{~ms}$ across all three stimuli types (incongruent, controls and fillers).

\section{Statistical analyses}

\section{Theta power}

Theta power time course was calculated using a Fast Fourier Transform in 20 ms steps using a window of $500 \mathrm{~ms}$ and a Hanning tapper that included 3 cycles of the central frequency of the 4$8 \mathrm{~Hz}$ band of interest. Data were baseline corrected using the $-750 \mathrm{~ms}$ to $-250 \mathrm{~ms}$ period and transformed in $\mathrm{dB}$. Then data were averaged across trials for the different conditions and each participant. Theta power was averaged across the electrodes of interest $\mathrm{Fz}, \mathrm{FCz}$ and $\mathrm{Cz}$ and across the Theta band (4-8 Hz), obtaining a single time-course for each participant and condition.

We extracted the maximum Theta peak amplitude for each participant and condition in the time window between $50 \mathrm{~ms}$ and $600 \mathrm{~ms}$ post-stimulus from the average activity from electrodes $\mathrm{Fz}$, $\mathrm{FCz}$ and $\mathrm{Cz}$. This is the typical time window used for frontal-medial Theta analysis in the typical laboratory protocol (Castro, Soto-Faraco, Morís Fernández, \& Ruzzoli, 2018; Cavanagh, Zambrano-Vazquez, \& Allen, 2012; Morís Fernández, Torralba, \& Soto-Faraco, 2018) and it minimizes possible evoked effects due to image onset $(<50 \mathrm{~ms})$. We then calculated the average amplitude within an $80 \mathrm{~ms}$ window centred on the peak (from -40 to $+40 \mathrm{~ms}$ ) for each participant and condition. Finally, we compared the peak amplitude between control and incongruent conditions separately, by running a t-test (one-tailed; $\alpha=0.05$ ) of the hypothesis that peak amplitude was larger for incongruent than control images. An equivalent exploratory nonregistered analysis was run on the latency of the peak (see below). We choose to initially test our hypothesis on the Theta peak (instead of the average activity though the entire time window) in order to preserve subject- and condition- peak differences in the Theta band (Castro et al., 2018; see for example Gulbinaite, van Rijn, \& Cohen, 2014). 


\section{Memory test}

For the behavioral memory test, we expected that incongruent images would be remembered better compared to control images (Krebs et al., 2015). Therefore, we tested whether the groupaverage correct response in incongruent trials was larger than control trials using a paired t-test (one tail; $\alpha=0.05$ ).

\section{Exploratory Analyses}

\section{Theta Power}

From the Theta power time course as described above, we also analyzed the latency of the Theta peak, comparing the peak latency between control and incongruent conditions separately by a ttest (two-tailed; $\alpha=0.05$ ). We also included a non-registered analysis that, instead of the single peak, compared the theta time-course from $250 \mathrm{~ms}$ to $1000 \mathrm{~ms}$ (time window decided in order to avoid potential contamination due to stimulus onset evoked activity). A right-tailed paired t-test was run with an alpha level of 0.05 for each time point within the window. Multiple comparison correction following Guthrie \& Buchwald (1991) determined a threshold of 7 consecutive significant $(\mathrm{p}<0.05)$ points $(\varphi \sim=0.99$, number of time points $=38, \mathrm{~N}=27$, and Theta $=.05)$. The same interval was significant when correcting for the time window 0 to $1000 \mathrm{~ms}$, which determines a threshold of 10 consecutive significant points. The same analysis was run but averaging across participants for each item to run the correlation by items.

\section{Alpha power}

Beyond the pre-registered protocol, we also decided to explore the related hypothesis that conflict detection triggers a dynamic adjustment of cognitive control via attention re-orienting (Compton, Huber, Levinson, \& Zheutlin, 2012). This hypothesis also is derived from the theoretical framework of cognitive control mechanisms (Botvinick et al., 2001). According to this hypothesis, attention should be boosted right after incongruent images, compared to after controls. Similarly, as for Theta power, we calculated for each participant and trial the power between 8 and $14 \mathrm{~Hz}$ from the average activity from posterior electrodes (P3, Pz, P4, O1, O2). These electrodes where chosen given that the expectation that attention effects would be best reflected in posterior scalp activity. We calculated the difference of the Alpha power between incongruent and control condition for each participant. This difference was analyzed at group level by a righttailed t-test $(\alpha=0.05)$. As a signature of allocation of attention, we looked at the Alpha power ( 8 $-14 \mathrm{~Hz}$ ) after image presentation. Indeed, a decrement of occipito-parietal Alpha power has been associated with effective attention allocation (Compton et al., 2012). Similarly, as for the Theta power, we compared Alpha power after incongruent and control images in the time window 150 - $1000 \mathrm{~ms}$ in $20 \mathrm{~ms}$ steps, using a right-tailed t-test $(\alpha=0.05)$ of the hypothesis that Alpha power would be lower in incongruent than congruent conditions. However, the comparison turned out to be not significant after correction and will not be discussed any further.

\section{Memory test RTS}

Similarly to the pre-registered analysis regarding accuracy in the memory test, we also run an analysis on the group average response times (RTs). Only correct response RTs within $\pm 2 \mathrm{SD}$ 
around the individual mean were considered for the analysis. As we did not have a hypothesis on RTs we applied a two-tailed t-test $(\mathrm{p}<0.05)$.

\section{Item by item correlation between Theta power and memory performance}

We explored the possibility that some relationship existed between the Theta power modulation found in the EEG analysis and the behavioural memory effect. Specifically, we tested whether images that produced a stronger Theta power response (raw Theta power averaged across participants per each image) were remembered better (i.e., higher accuracy and lower reaction times one week later) by an item-by-item correlation. To estimate Theta power for this correlation, we calculated the average raw Theta power in the window $370-710 \mathrm{~ms}$, that had resulted significant at group level when comparing the surrealistic and control conditions. We performed the same analyses at a participant level, but in this case, instead of the raw Theta power, we calculated the average difference between surrealistic and control images in the same time window as before, and then ran the correlation with memory performance.

\section{Results}

As per the predictions from the pre-registered hypothesis, our focus was on the Theta power (4-8 $\mathrm{Hz}$ ) at frontal-medial locations (reflected in $\mathrm{Fz}, \mathrm{Cz}, \mathrm{FCz}$ ). We extracted the maximum Theta peak amplitude for each participant and condition in the time window between $50 \mathrm{~ms}$ and $600 \mathrm{~ms}$ after image presentation. The difference in the peak amplitude (average amplitude $\pm 40 \mathrm{~ms}$ around the peak) in the Theta band between incongruent $(1.49 \pm 1.12)$ and control $(1.33 \pm 1.33)$ conditions was not significant $\left[\mathrm{t}(25)=0.69 ; \mathrm{p}=0.246 ; \mathrm{d}_{\mathrm{z}}=0.135\right]$. However, the latency of the Theta peaks was different between conditions [incongruent $0.26 \mathrm{~s} \pm 0.12$; control $0.20 \mathrm{~s} \pm 0.09 ; \mathrm{t}(25)=2.09$; $\left.\mathrm{p}=0.047 ; \mathrm{d}_{\mathrm{z}}=0.409\right]$, which we interpreted as a difference in time evolution of the frontal-medial Theta responses between conditions. We therefore decided to run a non-registered analysis on the frontal-medial Theta power, but across time. To do this, we compared frontal-medial Theta amplitude in response to incongruent and control images in the time window $250-1000 \mathrm{~ms}$ in 20 ms steps, using a right-tailed t-test $(\alpha=0.05)$ of the hypothesis that Theta power would be larger in incongruent than congruent conditions over time. Multiple comparison correction following Guthrie \& Buchwald (1991) determined a threshold of 7 consecutive significant $(\mathrm{p}<0.05)$ points $(\varphi \sim=0.99$, number of time points $=38, \mathrm{~N}=27$, and Theta $=.05)$. We found significant differences in frontal-medial Theta power in a $340 \mathrm{~ms}$ time window (between 370 and $710 \mathrm{~ms}$ ), with 18 consecutive significant points, revealing that looking at incongruent images was associated to a higher Theta power compared to controls (see Figure 2). It is important to note that the two analyses (the original pre-registered analysis on the Theta peak and the Theta power time evolution) address different aspects of the Theta response, but under the same (pre-registered) hypothesis that surrealistic images would elicit stronger Theta power compared to controls.

As we were also interested in the memorability of the surrealistic images compared to control, we invited participants to come back to the lab one week after the EEG recordings and perform a memory test: they were asked to choose which of two images presented side-by-side they remembered seeing during the EEG session (two-alternative forced-choice -2AFC- task)(Figure 1B). As expected, incongruent images were remembered more often (hit $=84 \% \pm 9 \%$ ) compared to control images (hit $=71 \% \pm 13 \%$ ) $\left[\mathrm{t}(26)=6.77 ; \mathrm{p}=3 \mathrm{e}^{-07}, \mathrm{~d}_{\mathrm{z}}=1.3\right]$. Mean reaction time (RT) for incongruent images $(2.2 \mathrm{~s} \pm 0.60)$ was faster than for control images $(2.4 \pm 0.8)[\mathrm{t}(26)=-3.28$; $\left.\mathrm{p}=0.00291, \mathrm{~d}_{\mathrm{z}}=-0.63\right]$ (Figure 3).

Given the positive results on both the Theta power modulation and the memory test, we decided to run an item-by-item correlation between the two measurements (exploratory analysis). We found a 
small but significant correlation at item-by-item level between Theta power and memory accuracy $(\mathrm{t}=2.180, \mathrm{df}=198, \mathrm{p}$-value $=0.030,95 \% \mathrm{CI}=[0.150 .29], \mathrm{r}=0.15)$. This correlation suggests that the images that evoked higher frontal-medial Theta power responses are the ones more often remembered one week after the EEG phase (Figure 4). At participant level, we found no correlation between the Theta power difference and accuracy $(\mathrm{t}=-1.888, \mathrm{df}=25, \mathrm{p}$-value $=0.070,95 \% \mathrm{CI}=$ [-0.65 0.03], $\mathrm{r}=-0.35)$. This means that participants with stronger Theta modulation are not necessarily the ones who will remember images more successfully. The correlation between Theta power and the reaction times was not significant neither across items $(\mathrm{t}=-0.122, \mathrm{df}=198, \mathrm{p}$-value $=0.903,95 \% \mathrm{CI}=[-0.150 .13], \mathrm{r}=-0.009)$ nor participants $(\mathrm{t}=-1.45, \mathrm{df}=25, \mathrm{p}$-value $=0.158,95 \%$ $\mathrm{CI}=[-0.600 .11], \mathrm{r}=-0.28)$.

Figures 3 and 4 about here

\section{Discussion}

We tested the hypothesis that surrealistic imagery, a form of creative communication in arts and advertising, triggers cognitive conflict responses in the brain. We also tested the related assumption that episodic memory for images with surrealistic content would be superior to other, congruent images. We based our predictions on the conflict monitoring theory (Botvinick et al., 2001, 2004; Botvinick \& Cohen, 2014; Inzlicht et al., 2017; Shenhav et al., 2013, 2016), which allowed us to be specific regarding the expected results (see pre-registration https://osf.io/wftd7/). We measured frontal-medial Theta EEG activity, a reliable neuromarker of cognitive conflict (Cavanagh, Figueroa, et al., 2012), following the presentation of surrealistic images selected from media advertisement compared with visually similar but realistic imagery. As predicted, albeit with a non-registered analysis pipeline, we found that surrealistic advertising imagery not only generated stronger Theta activity at frontal-medial scalp locations compared to control images, it also produced higher recognition rates in a memory task delivered one week after the exposure, a key behavioral metric of advertising effectiveness (Keller, 1987). Furthermore, item-by-item Theta power and subsequent memory recognition for the corresponding image showed a correlation. This suggests that the stronger the Theta response an image elicits in the brain, the more likely it will be remembered, albeit the effect size of this correlation was small.

Surrealistic imagery generates a pattern of brain activation (i.e., frontal-medial Theta power increment) similar to the one seen in the conflict studies that have grounded cognitive control theories (Botvinick et al., 2004; Shenhav et al., 2013). Based on this, we suggest that surrealistic imagery generates conflict at a cognitive level and can be accounted for by these theories.

Consistent with our account, Mostafa (Mostafa, 2013) used fMRI to measure BOLD responses during passive viewing of advertisements, exploring which brain areas were activated by surrealistic imagery. He found that surrealistic imagery elicited greater activation in the ACC (among several other areas), compared to other images. Mostafa also suggested that the violation of expectations induced by surrealistic images may favor later recall, although he did not test memory recall explicitly. Here, we were able to confirm this assumption: surrealistic images are remembered better than other kinds of images. This hypothesis is implicit in real-world advertisement, although previous cognitive neuroscience findings show that, indeed, incongruent items in a scene are memorable. For example, Krebs and collaborators (2015), explored the impact of cognitive conflict on memory using a modified version of the Stroop task (i.e., the face- 
word Stroop task), showing that faces associated with incongruent stimuli were remembered more. In another study, by Friedman (1979), participants noticed only the changes that had been made to objects that were unexpected based on the surrounding scene (e.g., a coffeemaker shown in a farmyard, compared to a kitchen). Finally, Homer \& Kahle (1986) found that participants who were exposed to surrealistic ads were better at free-recall, compared to a control group exposed to non-surrealistic ads. In line with the mentioned studies, the present study showed that incongruent images were remembered better than controls one week later. Remarkably, frontalmedial Theta responses to images correlated with memory performance on image-by-image basis. This finding suggests a potential link between brain mechanisms of cognitive control and encoding into episodic memory. This link can find application in the context of creative communication strategies, grounding a long-standing assumption in advertising through surrealistic images. Our results provide a viable experimental procedure and proof-of-concept data that helps bridge the gap between the scientific and creative approaches and uncover new insights into the relationship between cognitive conflict and creativity.

The type of conflict addressed in the present study is generated by incongruence among visual properties of a stimulus, independently of conflict at the response level. The original version of the conflict monitoring theory proposed that conflict could arise at various stages of information processing, from perceptual representation to stimulus categorization and response selection (Botvinick et al., 2001). Yet, to date, most of the studies related to frontal-medial Theta power and cognitive control struggle to disambiguate between response conflict and stimulus conflict (Botvinick \& Cohen, 2014). Given the nature of our paradigm, pure stimulus conflict was relevant, and we only analyzed the response from image onset, avoiding response preparation or motor activity using a delayed response protocol and random assignment of response sides. Our results suggest that taking a step away from typical laboratory tasks can lead to discoveries that are of interest to the theory itself, in addition to real-life applications.

The idea (and the evidence provided here) that cognitive control mechanisms might go in line with the processing of surrealistic images establishes a fruitful connection between neurocognitive theories and creative communication strategies, such as those applied in marketing. For example, although it is often assumed that humans avoid conflict because it is energetically costly (physical and/or mental effort) (Dreisbach \& Fischer, 2012, 2015), one suggestive possibility is that conflict resolution could also act as internal reward (Inzlicht et al., 2017; Kurzban, 2016). Recently, Inzlicht et al. (2017) proposed a computational model in which effort can be considered both a cost and an added value which works as intrinsic reward and motivation. Incongruent images may require extra processing (i.e., effort) to be interpreted, which could have positive consequences (i.e. stronger memory). Therefore, extending the proposal by Inzlicht, et al. (2017), the creative communication strategy typically used in advertisement and arts not only elicits cognitive conflict and increased memory, as our data suggest, but might also be seen as an intrinsic reward, potentially linked to positive subjective experience (Chetverikov \& Kristjánsson, 2016; Van de Cruys \& Wagemans, 2011).

So far, we have only considered the interpretation that surrealistic advertising images elicit cognitive conflict; but, alternatives should be explored. Theta power enhancement at frontalmedial locations is considered a reliable measure of cognitive conflict, as demonstrated by the classical experimental paradigms (i.e., Stroop, Flanker, Simon tasks) of cognitive control (Cavanagh, Zambrano-Vazquez, et al., 2012; Macleod, 1991). However, Theta power enhancement has also been associated with novelty, errors, or even feedback on errors (Cavanagh, Zambrano-Vazquez, et al., 2012). Because the task during the EEG recordings was orthogonal to the congruency manipulation, it is doubtful that the Theta effects could be attributed 
to participant's errors or negative feedback. In fact, no errors could be possible, nor feedback was provided. In addition, an element of increased novelty is necessarily conflated with surrealism, and therefore its contribution to our main finding should be considered. Indeed, we decided to select the incongruent images based on originality and synthesis dimensions of ads divergence as by Smith et al. (Smith et al., 2007), which both imply novelty to some extent.

In conclusion, this study provides an original piece of evidence regarding the power of surrealistic images to generate cognitive conflict in the brain and to boost memory, as was the original spirit of the surrealist movement at its birth: "The image is a pure creation of the mind. It cannot be born from a comparison but from a juxtaposition of two more or less distant realities. The more the relationship between the two juxtaposed realities is distant and true, the stronger the image will be - the greater its emotional power and poetic reality..." Pierre Reverdy, 1918 in Manifesto of Surrealism (Breton, 1985). 


\section{Conflict of Interest}

The authors declare no competing financial interests.

\section{Acknowledgements (65)}

This research was supported by the Ministerio de Economia y Competitividad (PSI2016-75558P AEI/FEDER), AGAUR Generalitat de Catalunya (2017 SGR 1545), and the European Research Council (PoC- 727595). M.R. was supported by a Marie Skłodowska-Curie fellowship (Ctrl Code - 794649 - H2020-MSCA-IF-2017). We wish to thank Marc Lluís Vives for his comments to a preliminary version of the manuscript and the discussions about the topic.

\section{Author contributions}

M.R. designed the experiment, helped in data collection, interpreted the results and wrote the manuscript. A.M.G. designed the experiment, selected the images used in the study, collected and analysed the data, interpreted the results, drafted the manuscript. L.M.F. designed the experiments and data analyses, wrote the codes for the EEG data analysis, analyzed the data, interpreted the results, and drafted the manuscript. S.S.F. designed the idea and the experiment, interpreted the results and wrote the manuscript. 


\section{Figures Captions}

Fig 1: A. Schematic representation of a trial during the EEG recording session. A trial started with a $1500 \mathrm{~ms}$ fixation (loading wheel), followed by the target image (incongruent, control or filler) presented for $2000 \mathrm{~ms}$. A response screen prompted the participants to choose whether they thought the presented image was directed to females or males (the position of the icons was chosen randomly at each trial) and lasted till the response was provided. The next trial started after a variable inter-trial interval (ITI) randomly jittered between 800 and $1000 \mathrm{~ms}$. A representation of the 16 EEG electrodes montage is also provided. B. Schematic representation of the memory test performed about one week after the EEG test. Participants were asked to fixate on a fixation presented for $1000 \mathrm{~ms}$, before the image pair appearance. They had to choose which of the presented images (both incongruent or control) had been presented the week before during the EEG test.

Fig 2: Difference in Theta power $(4-8 \mathrm{~Hz})$ between incongruent and control images calculated from mid-frontal electrodes $(\mathrm{Fz}, \mathrm{Cz}, \mathrm{FCz})$ as a function time, where 0 signals image onset. The interval $(370-710 \mathrm{~ms})$ of significant difference (Theta power for incongruent images was significantly higher than for controls) is bounded within dashed vertical lines. The shaded area represents the standard error of the mean.

Fig 3: Results from the memory test in terms of accuracy (A) and reaction times (B) for incongruent and control images, and for the difference between the two. Each dot represents a participant, bars show the group average and the standard error of the mean, respectively.

Fig 4: Image-by-image correlation between induced Theta power (x-axis) and the proportion of correct responses (y-axis) at the memory test. Least squares linear fit and its confidence interval are shown with the dashed line and shaded area, respectively. 


\section{Bibliography}

Botvinick, M. M., Braver, T. S., Barch, D. M., Carter, C. S., \& Cohen, J. D. (2001). Conflict monitoring and cognitive control. Psychological Review, 108(3), 624-652. https://doi.org/10.1037/0033-295X.108.3.624

Botvinick, M. M., \& Cohen, J. D. (2014). The computational and neural basis of cognitive control: Charted territory and new frontiers. Cognitive Science, 38(6), 1249-1285. https://doi.org/10.1111/cogs.12126

Botvinick, M. M., Cohen, J. D., \& Carter, C. S. (2004). Conflict monitoring and anterior cingulate cortex: An update. Trends in Cognitive Sciences, 8(12), 539-546. https://doi.org/10.1016/j.tics.2004.10.003

Brady, T. F., Konkle, T., \& Alvarez, G. A. (2011). A review of visual memory capacity: Beyond individual items and toward structured representations. Journal of Vision, 11(5), 4-4. https://doi.org/10.1167/11.5.4

Brainard, D. H. (1997). The Psychophysics Toolbox. Spatial Vision, 10(4), 433-436.

Braver, T. S., Barch, D. M., \& Gray, J. R. (2001). Anterior Cingulate Cortex and Response Conflict: Effects of Frequency, Inhibition and Errors. Cerebral Cortex, 11, 825-836.

Breton, A. (1985). Manifestes du surréalisme. Gallimard.

Castro, L., Soto-Faraco, S., Morís Fernández, L., \& Ruzzoli, M. (2018). The breakdown of the Simon effect in cross-modal contexts: EEG evidence. European Journal of Neuroscience, 47(7). https://doi.org/10.1111/ejn.13882

Cavanagh, J., Figueroa, C. M., Cohen, M. X., \& Frank, M. J. (2012). Frontal theta reflects uncertainty and unexpectedness during exploration and exploitation. Cerebral Cortex, 22(11), 2575-2586. https://doi.org/10.1093/cercor/bhr332

Cavanagh, Zambrano-Vazquez, L., \& Allen, J. J. B. J. J. B. (2012). Theta lingua franca: A common mid-frontal substrate for action monitoring processes. Psychophysiology, 49(2), 220-238. https://doi.org/10.1111/j.1469-8986.2011.01293.x

Chetverikov, A., \& Kristjánsson, Á. (2016). On the joys of perceiving: Affect as feedback for perceptual predictions. Acta Psychologica, 169, 1-10.

https://doi.org/10.1016/j.actpsy.2016.05.005

Cohen, M. X. (2014). A neural microcircuit for cognitive conflict detection and signaling. Trends in Neurosciences, 37(9), 480-490. https://doi.org/10.1016/j.tins.2014.06.004

Compton, R. J., Huber, E., Levinson, A. R., \& Zheutlin, A. (2012). Is 'conflict adaptation' driven by conflict? Behavioral and EEG evidence for the underappreciated role of congruent trials. Psychophysiology, 49(5), 583-589. https://doi.org/10.1111/j.14698986.2012.01354.x

Dreisbach, G., \& Fischer, R. (2012). Conflicts as aversive signals. Brain and Cognition, 78(2), 94-98. https://doi.org/10.1016/J.BANDC.2011.12.003

Dreisbach, G., \& Fischer, R. (2015). Conflicts as Aversive Signals for Control Adaptation. Current Directions in Psychological Science, 24(4), 255-260. https://doi.org/10.1177/0963721415569569

Dzulkifli, M. A., \& Mustafar, M. F. (2013). The influence of colour on memory performance: a review. The Malaysian Journal of Medical Sciences : MJMS, 20(2), 3-9. Retrieved from http://www.ncbi.nlm.nih.gov/pubmed/23983571

Eriksen, B. A., \& Eriksen, C. W. (1974). Effects of noise letters upon the identification of a target letter in a nonsearch task. Perception \& Psychophysics, 16(1), 143-149. https://doi.org/10.3758/BF03203267 
Friedman, A. (1979). Framing pictures: the role of knowledge in automatized encoding and memory for gist. Journal of Experimental Psychology. General, 108(3), 316-355. Retrieved from http://www.ncbi.nlm.nih.gov/pubmed/528908

Gulbinaite, R., van Rijn, H., \& Cohen, M. X. (2014). Fronto-parietal network oscillations reveal relationship between working memory capacity and cognitive control. Frontiers in Human Neuroscience, 8, 761. https://doi.org/10.3389/fnhum.2014.00761

Guthrie, D., \& Buchwald, J. S. (1991). Significance Testing of Difference Potentials. Psychophysiology, Vol. 28, pp. 240-244. https://doi.org/10.1111/j.14698986.1991.tb00417.x

Homer, P. M., \& Kahle, L. R. (1986). A social adaptation explanation of the effects of surrealism on advertising. Journal of Advertising, 15(2), 50-60. https://doi.org/10.1080/00913367.1986.10673005

Inzlicht, M., Shenhav, A., \& Olivola, C. Y. (2017). The Effort Paradox: Effort is Both Costly and Valued. SSRN Electronic Journal. https://doi.org/10.2139/ssrn.3069591

Keller, K. L. (1987). Memory Factors in Advertising: The Effect of Advertising Retrieval Cues on Brand Evaluations. Journal of Consumer Research, 14(3), 316. https://doi.org/10.1086/209116

Kerns, J. G., Cohen, J. D., MacDonald, A. W., Cho, R. Y., Stenger, V. A., \& Carter, C. S. (2004). Anterior cingulate conflict monitoring and adjustments in control. Science (New York, N.Y.), 303(5660), 1023-1026. https://doi.org/10.1126/science.1089910

Krebs, R. M., Boehler, C. N., De Belder, M., \& Egner, T. (2015). Neural conflict-control mechanisms improve memory for target stimuli. Cerebral Cortex, 25(3), 833-843. https://doi.org/10.1093/cercor/bht283

Kurzban, R. (2016). The sense of effort. Current Opinion in Psychology, 7, 67-70. https://doi.org/10.1016/j.copsyc.2015.08.003

Lee, E. ju, \& Schumann, D. W. (2004). Explaining the special case of incongruity in advertising: Combining classic theoretical approaches. Marketing Theory, 4(1-2), 59-90. https://doi.org/10.1177/1470593104044087

Macleod, C. M. (1991). Psychological Bulletin Half a Century of Research on the Stroop Effect: An Integrative Review. In Psychological Association, Inc. 199 l (Vol. 109). Retrieved from http://melaniestefan.net/MacLeod1991.pdf

Matusz, P. J., Dikker, S., Huth, A. G., \& Perrodin, C. (2019). Are We Ready for Real-world Neuroscience? Journal of Cognitive Neuroscience, 31(3), 327-338. https://doi.org/10.1162/jocn_e_01276

Morís Fernández, L., Torralba, M., \& Soto-Faraco, S. (2018). Theta oscillations reflect conflict processing in the perception of the McGurk illusion. European Journal of Neuroscience, 48(7), 2630-2641. https://doi.org/10.1111/ejn.13804

Mostafa, M. M. (2013). The persistence of memory: An fMRI investigation of the brain processing of Surrealistic imagery in advertising. Journal of Marketing Communications, 19(5), 341-359. https://doi.org/10.1080/13527266.2011.653688

Oostenveld, R., Fries, P., Maris, E., \& Schoffelen, J.-M. (2011). FieldTrip: Open source software for advanced analysis of MEG, EEG, and invasive electrophysiological data. Computational Intelligence and Neuroscience, 2011, 156869. https://doi.org/10.1155/2011/156869

Qualtrics. (n.d.). Retrieved from https://www.qualtrics.com

Shenhav, A., Botvinick, M. M., \& Cohen, J. D. (2013). The Expected Value of Control: An Integrative Theory of Anterior Cingulate Cortex Function. Neuron, 79(2), 217-240. 
https://doi.org/10.1016/j.neuron.2013.07.007

Shenhav, A., Cohen, J. D., \& Botvinick, M. M. (2016). Dorsal anterior cingulate cortex and the value of control. Nature Neuroscience, 19(10), 1286-1291.

https://doi.org/10.1038/nn.4384

Simon, J. R., \& Small, A. M. (1969). Processing auditory information: Interference from an irrelevant cue. Journal of Applied Psychology, 53(5), 433-435.

https://doi.org/10.1037/h0028034

Smith, R. E., MacKenzie, S. B., Yang, X., Buchholz, L. M., Darley, W. K., Smith, R. E., ... MacKenzie, S. B. (2007). Modeling the Determinants and Effects of Creativity in Advertising. Marketing Science, 26(6), 819-833. https://doi.org/10.1287/mksc.1070.0272

Soto-Faraco, S., Kvasova, D., Biau, E., Ikumi, N., Ruzzoli, M., Morís-Fernández, L., \& Torralba, M. (2019). Multisensory Interactions in the Real World. In James T. Enns (Ed.), Elements in Perception. https://doi.org/10.1017/9781108578738

Stroop, J. R. (1935). Studies of interference in serial verbal reactions. Journal of Experimental Psychology, 18(6), 643-662. https://doi.org/10.1037/h0054651

Van de Cruys, S., \& Wagemans, J. (2011). Putting Reward in Art: A Tentative Prediction Error Account of Visual Art. I-Perception, 2(9), 1035-1062. https://doi.org/10.1068/i0466aap 


\section{A. EEG test}

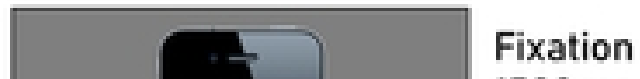

$1500 \mathrm{~ms}$

Image $2000 \mathrm{~ms}$

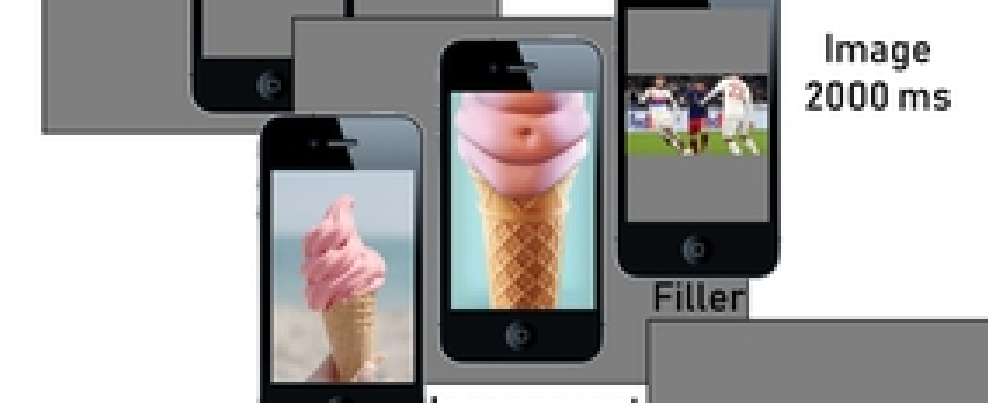

Incongruent Control
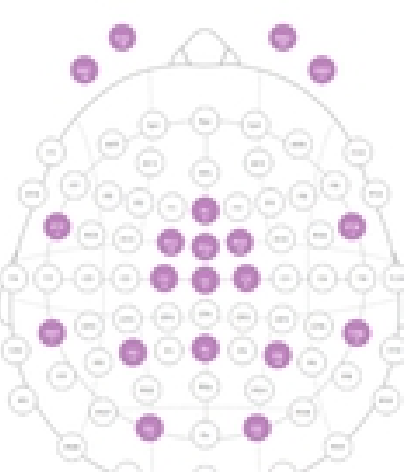

EEG montage

Figure 1

\section{B. Memory test}

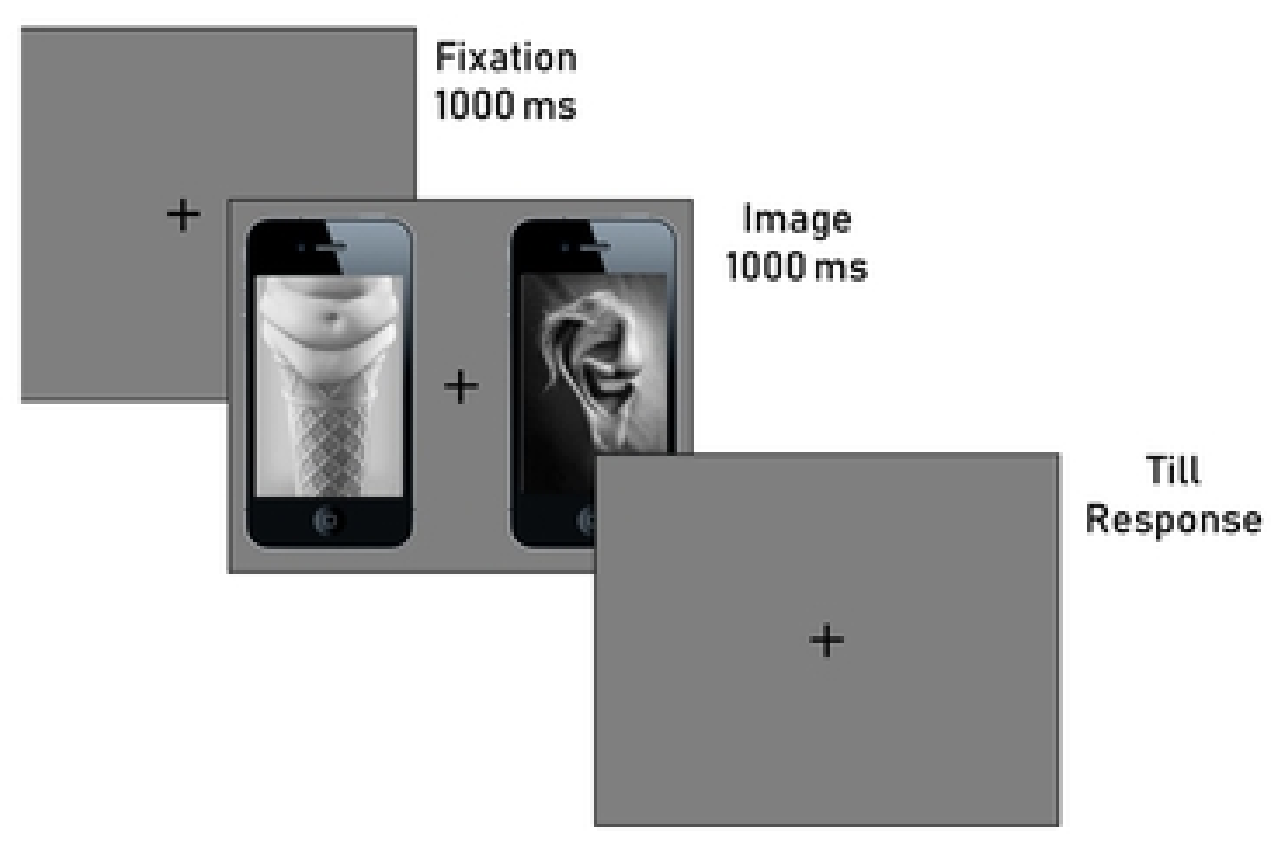

Fixation

$000 \mathrm{~ms}$

$800-1000 \mathrm{~ms}$

0

Till

Response

○

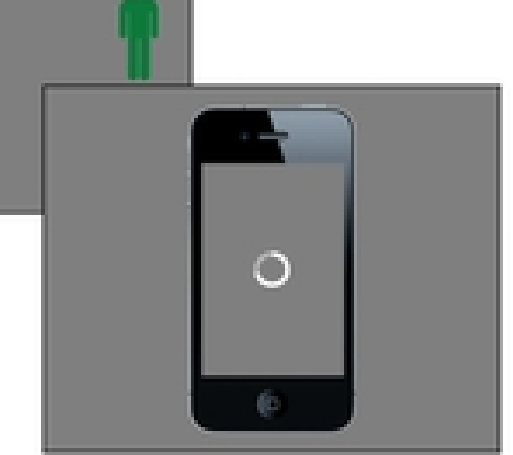



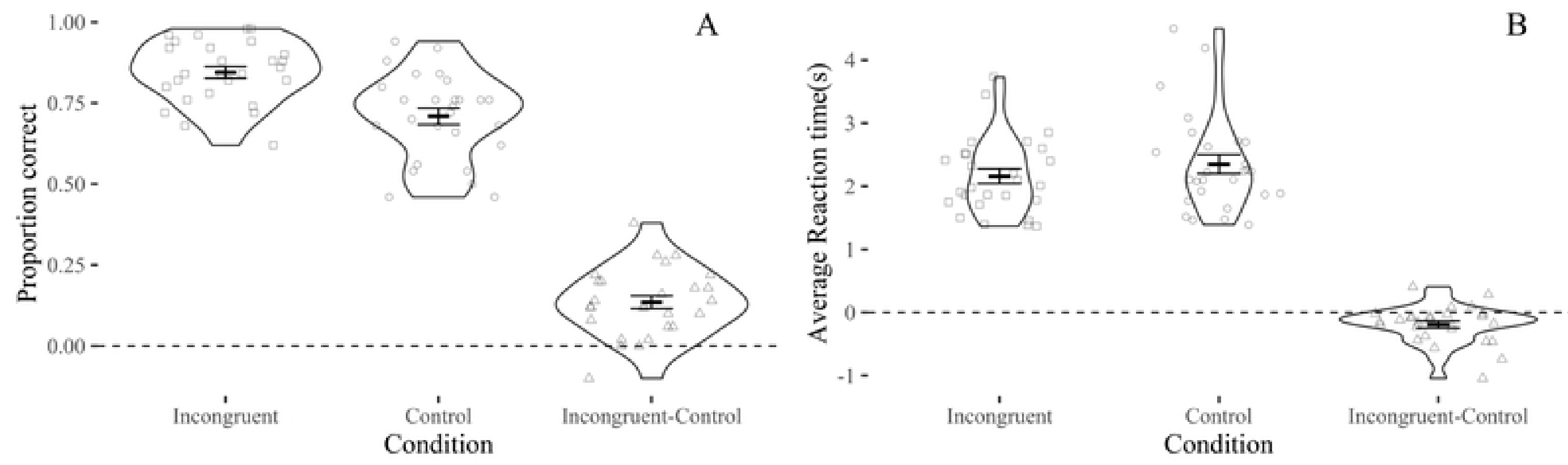

Figure 3 


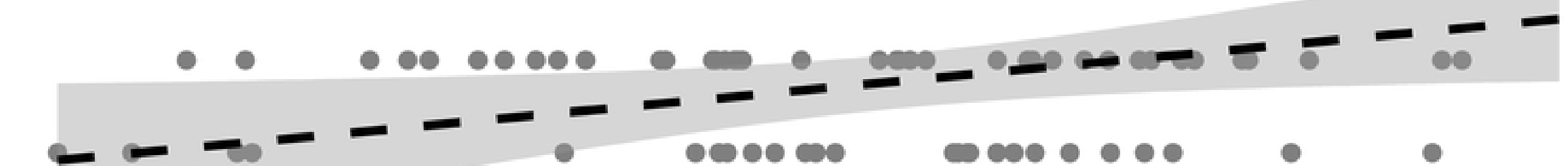

$0.4-$

$\bullet$

Figure 4 